\title{
Comparative analysis of His-bundle pacing and left bundle branch area pacing: acute and short-term results
}

\author{
Manuel Molina-Lerma ${ }^{1}$, Rosa Macias-Ruiz ${ }^{1}$, Pablo Sánchez-Millán² ${ }^{2}$ Juan Jiménez-Jáimez ${ }^{1}$, \\ Luis Tercedor ${ }^{1}$, Mercedes Cabrera ${ }^{1}$, Javier Ramos ${ }^{1}$, Guillermo Gutierrez Ballesteros ${ }^{1}$, \\ María Molina-Jiménez ${ }^{1}$, and Miguel Alvarez ${ }^{1}$ \\ ${ }^{1}$ Hospital Universitario Virgen de las Nieves \\ ${ }^{2}$ Virgen de las Nieves Universitary Hospital
}

June 12,2020

\begin{abstract}
Introduction: His-bundle pacing (HBP) and left bundle branch area pacing (LBBAP) are novel techniques that have demonstrated their efficacy in patients needing right ventricular pacing and in candidates for resynchronization therapy. The purpose of this study was to compare acute and short-term outcomes between HBP and LBBAP. Methods: Retrospective review of the success rate and change in QRS duration obtained by HBP and LBBAP in patients requiring anti-bradycardia pacing or cardiac resynchronization, evaluating the complications and pacing parameters at implantation and at 3 months. Results. The study included 90 procedures (46 HBP and $44 \mathrm{LBBAP}$ ) in 87 patients. Success was achieved in 77 procedures (85.6\%): 37 (80.4\%) in the HBP group and $40(90.9 \%)$ in the LBBAP group $(\mathrm{P}=.23)$. Paced QRS duration was shorter in the LBBAP patients $(109 \pm 8$ vs. $117 \pm 19 \mathrm{~ms} ; \mathrm{P}<.05)$, who had a lower threshold during implantation $(0.5 \mathrm{~V} / 0.5 \mathrm{~ms}$ vs. $1.5 \mathrm{~V} / 1 \mathrm{~ms} ; \mathrm{P}<.001)$ and at 3 months $(0.72 \mathrm{~V} / 0.5 \mathrm{~ms}$ vs. $1.75 \mathrm{~V} / 1 \mathrm{~ms} ; \mathrm{P}<.001)$ and an increased sensed R-wave amplitude. The fluoroscopy time was shorter in the LBBAP group (10 vs. 19 minutes; $\mathrm{P}<.001)$. The complication rate was similar $(6.8 \%$ vs. $12.8 \% ; \mathrm{P}=.48)$. Conclusion. In our experience, LBBAP obtains a similar success rate to that of HBP and achieves a shorter paced QRS duration, greater R-wave detection, lower threshold, and shorter fluoroscopy time, with a similar complication rate.
\end{abstract}

\section{Introduction}

Since its first description ${ }^{1}$, selective and non-selective His-bundle pacing (HBP) has proven to be a feasible and safe procedure, with morbidity and mortality outcomes comparable or superior to those of conventional endocardial right-ventricular pacing. ${ }^{2,3}$ It has also achieved excellent outcomes in candidates for cardiac resynchronization therapy (CRT).$^{4,5}$ Nevertheless, well-documented limitations of HBP include a long learning curve, higher energy requirement, greater radiological exposure, and the possible need for reintervention due to lead-related complications. ${ }^{6}$ In addition, the success rate of this procedure is lower in patients with interventricular conduction defects, because branches of the His-Punkinje system cannot be recruited when the block zone is more distal from the pacing point. ${ }^{7}$

Left bundle branch area pacing (LBBAP), in which the lead penetrates into the interventricular septum, is a feasible and safe alternative option in candidates for antibradycardia therapy (ABT) or CRT and has also obtained similar or better outcomes in comparison to conventional pacing. ${ }^{89}$ However, no studies have been published that compare results between these two techniques. The objective of the present study was to compare outcomes between HBP and LBBAP at implantation and at 3 months.

\section{Methods}

\section{Selection of patients}


A retrospective review was conducted of a prospectively studied cohort of consecutive patients scheduled for ABT and CRT who underwent cardiac device implantation by the same electrophysiologist. HBP alone was performed during the first study period (January through December 2018) and LBBAP alone during the second study period (January through December 2019). The study was approved by the local Ethics Committee.

\section{Implantation procedure}

A single cardiologist was responsible for implantation, while other operators participated at various stages of the procedure (venous cannulation, atrial lead placement).

Fluoroscopy-guided cannulation of the axillary vein was the venous access of choice, using a lumen-less 4-Fr lead (Select-Secure model $383069 \mathrm{~cm}$, Medtronic Inc., Minneapolis, MN) through a fixed preformed sheath (C315 HIS, Medtronic Inc.). A deflectable sheath (C304, Medtronic Inc.) was used when it proved impossible to record the His electrogram. The lead was connected to a digital recording system (Electrophysiology Lab System, Boston Scientific, MA) in a unipolar configuration to measure the intracavitary signal.

HBP was performed as described in the literature. ${ }^{10}$ In brief, rotation and advance-retreat maneuvers were used to find the His bundle electrogram with lowest atrial signal amplitude, evaluating the selective and nonselective HBP with the lowest possible threshold. The bundle block correction threshold was also considered in patients with a wide baseline QRS.

After localization of the His electrogram in the LBBAP procedure, the sheath was apically advanced 1-2 $\mathrm{cm}$; next, if a paced QRS with "W" morphology was obtained in lead V1, the electrode was inserted into the septum with 5-6 clockwise turns until the notch in the paced QRS complex migrated to the end of the QRS wave (or disappeared) and the QRS width narrowed (Figure 1). The depth at which the lead was affixed in the interventricular septum was guided by the impedance values and target QRS obtained. Recording of the left bundle branch potential was not an objective. The fluoroscopy time included the time for the axillary puncture and for placing all of the leads.

The QRS complex was recorded before and after implantation by another operator using a digital recording system at a speed of $100 \mathrm{~mm} / \mathrm{s}$. The QRS duration was measured from QRS onset in the 12 leads when an isoelectric interval was observed between pacing stimulus and QRS onset or from stimulus to the end of the QRS when it was not. Pacing parameters (threshold, sensed R-wave amplitude, impedance) were compared between implantation and 3 months post-implantation.

The procedure was considered successful when a baseline QRS of $<120 \mathrm{~ms}$ was achieved in HPB, as recommended by the Multicenter HBP Collaborative Working Group ${ }^{1}$, and when a QRS width [?] $130 \mathrm{~ms}$ was obtained in LBBAP. ${ }^{12}$ In patients with bundle branch block (BBB), HPB and LBBAP procedures were considered successful when the paced QRS narrowed by [?]20\% or to a QRS width of $<130 \mathrm{~ms}{ }^{13}$. Accepted pacing parameters were a pacing threshold of [?] $3.5 \mathrm{~V}$, an R-wave amplitude [?] $0.8 \mathrm{mV}$, and a pulse width of $1 \mathrm{~ms}$ with HBP and $0.5 \mathrm{~ms}$ with LBBAP.

\section{Statistical analysis}

Quantitative variables were expressed as means with standard deviation if they followed a normal distribution and medians (interquartile range) if they did not, and qualitative variables were expressed as percentages. Qualitative variables were compared with the $\chi^{2}$ test or Fisher's exact test and quantitative variables with the Student's t-test or Mann-Whitney test. $\mathrm{P}<0.05$ was considered significant. The SPSSß statistical program (version 21.0, Chicago, USA) was used for data analyses.

In successful cases, baseline and paced QRS duration and pacing parameters were compared between implantation and 3 months post-implantation. We also compared paced QRS complex duration values as a function of the baseline duration and between procedures in patients with BBB at baseline. The degree of variation in the QRS complex under different circumstances was analyzed by using the QRS narrowing index (QCI): ([paced QRS duration minus baseline QRS duration]) divided by baseline QRS duration) x $100 .{ }^{14}$ 


\section{Results}

Ninety procedures were performed in 87 patients (median age 76 years; $61.1 \%$ male): 46 (51.1\%) underwent HBP and $44(48.9 \%)$ underwent LBBAP. One patient underwent two HBP implantations and two patients underwent LBBAP after an initial HBP procedure due to lead-related complications. Table 1 exhibits the baseline characteristics of the patients. There were no significant differences between HBP and LBBAP groups in hypertension (HT) $(P<.01)$, structural heart disease $(P<.05)$, or sinus atrial rhythm $(P$ $=.05)$. All patients with wide baseline QRS $(\mathrm{n}=49)$ had a QRS duration of [?] $130 \mathrm{~ms}$. Left bundle branch potential was recorded in $7(15.9 \%)$ of LBBAP procedures.

A successful outcome was obtained in 85\% $(77 / 90)$ of the implantations, $80.4 \%(\mathrm{n}=37)$ in the HBP group and $90.9 \%(\mathrm{n}=40)$ in the LBBAP group $(P=.23)$. Among the thirteen failures, four were in the LBBAP group and nine in the HBP group. The interventricular septum could not be penetrated in all patients in the LBBAP group. In the HBP group, the His electrogram was not recorded in five, there was no QRS reduction in two indicated for CRT, the paced QRS width was $>130 \mathrm{~ms}$ (baseline $<120 \mathrm{~ms}$ ) in one, and effective capture was not achieved in the other. Conventional CRT was performed in seven cases of failure and also in six cases of anti-bradycardia pacing, with the lead being placed in the right ventricle outflow tract in five and kept in the HBP zone in one (QRS $136 \mathrm{~ms}$ ). The fluoroscopy time was shorter for LBBAP than for HBP (10 vs. 17 min, $P<.001)$.

In the univariate analysis, a successful outcome was related to age, sex, baseline QRS width, baseline LVEF, and presence of a previous device; none was retained as an independent predictor, but the presence of a previous device was associated with a lower success rate (odds ratio (OR) 5.9; 95\% confidence interval (CI) $0.85-42.2 ; P=.072)$.

Similar complication rates were recorded at the three-month follow-up (including complications before hospital discharge) between the HBP group $(12.8 \%, \mathrm{n}=6)$ and the LBBAP group $(6.8 \%, \mathrm{n}=3)(P=.48)$. There were three non-lead-related complications, one in the HBP group (pocket infection) and two in the LBBAP group (subacute pericardial effusion and hematoma). Lead-related complications were more frequent in the HBP group ( $10.6 \%$ vs. $2.3 \% ; P=.2$ ), with two losses of capture, one displacement, and two significant increases in pacing threshold; there was only one displacement in the LBBAP group (in a patient who had already suffered HBP displacement).

\section{Comparative analysis of HBP and LBBAP (Table 3)}

Non-selective HBP was obtained in $28(75.7 \%)$ of the 37 patients in the HBP group and selective HBP in 9 $(24.3 \%)$. The utilization of a deflectable sheath was more frequent in the HBP group. The fluoroscopy time (excluding cases of reintervention) was longer in the HBP group, regardless of the number of leads implanted. There was no significant difference in success rate between HBP and LBBAP groups among patients with narrow baseline QRS (HBP $82.4 \%$ vs. LBBAP $100 \% ; P=.23$ ) or among those with a wide QRS (HBP $79.3 \%$ vs. LBBAP $86.2 \% ; P=.73)$. The paced QRS duration was shorter in the LBBAP group $(109+-8$ vs. $117+-19 \mathrm{~ms} ; P<.05)$.

At implantation, the pacing threshold was lower and the sensed R-wave amplitude was greater in the LBBAP group, and these differences with the LBBAP group persisted at three months. Threshold and amplitude were increased in both groups at three months versus baseline, and the difference reached statistical significance in the LBBAP group (Figure 3).

\section{Progress of QRS complex width}

In the 77 successful cases (29 with narrow baseline QRS and 48 with wide baseline QRS), the mean QRS width was significantly reducedversus baseline $(141.3+-41$ vs. $112.7+-15.3 \mathrm{~ms} ; P<.001)$. In the successful cases with a baseline QRS of $<120 \mathrm{~ms}$ (14 in HBP and 15 in LBBAP), the paced QRS was significantly increased $(97.3+-7.1$ vs. $105.5+-10.3 \mathrm{~ms} ; P<.001)$ and the mean QCI was $8.7+-10.5 \%$ (range, $0-43.4 \%)$, with a slightly lower increase in the HBP group $(5.2+-10.2 \%$ vs. $12+-10 \% ; P=.08)$. Paced QRS width was prolonged in 19 patients, 15 in the LBBAP group and 4 in the HBP group; a QCI of 0 was obtained 
in the other 10 patients in the HBP group (7 cases of selective and 3 of non-selective HBP). Paced QRS remained at [?] $120 \mathrm{~ms}$ and $<130 \mathrm{~m}$ in only three patients (1 LBBAP and $2 \mathrm{HBP}$ ). Selective HBP obtained better results than non-selective HBP or LBBAP (Figure 4). In the 48 successful cases with baseline QRS of [?] $130 \mathrm{~ms}$ (25 in LBBAP and 23 in HBP), 23 had right bundle branch block (RBBB), 19 had left bundle branch block (LBBB), and 6 had QRS paced by a previously implanted device. The QCI was related to age, baseline QRS width, baseline LVEF, presence of LBBB or RBBB, and the technique (HBP or LBBAP). In the multiple linear regression, only baseline QRS width ( $\mathrm{OR}-3.4,95 \% \mathrm{CI}-0.32$ to $-0.08 ; P=.001)$ and LBBAP technique (OR 3.8, 95\% CI 4.4 to 14.5; $P<.001$ ) were retained as independent predictors. The reduction in QCI was greater with LBBAP than with $\mathrm{HBP}$ and even greater than with conventional CRT in failed cases (Figure 5).

\section{Bundle branch block}

There were 50 patients with BBB, 27 with RBBB, and 23 with LBBB. The overall success rate was $84 \%$ $(\mathrm{n}=42)$, with no significant difference $(P=.25)$ between $\operatorname{HBP}(76.9 \%)$ and LBBAP $(91.7 \%)$ groups. QRS narrowing was achieved in all patients with $\mathrm{BBB}$ except for two patients with no change in QRS width. A paced QRS $>120 \mathrm{~ms}$ was more frequent in the HBP group (60\% vs. $18.2 \% ; P<.01)$.

Among the patients with LBBB (12 in HBP and 11 in LBBAP), the success rate was close-to-significantly higher in the LBBAP group $(100 \%$ vs. $66.7 \% ; P=.09)$. Among successful cases, the paced QRS width was lower in the LBBAP group $(112+-9$ vs. $127+-26 \mathrm{~ms} ; P=.16)$, although the difference did not reach statistical significance, and the QCI (QRS narrowing) was similar between HBP $(-32.2+-13 \%)$ and LBBAP $(-34.8+-4.8 \%)$ groups $(P=.59)$. Among the patients with RBBB (14 in HBP and 13 in LBBAP), the success rate did not differ between HBP $(85.7 \%)$ and LBBAP $(84.6 \%)$ groups $(P=1)$, but the paced QRS width was lower $(106+-7$ vs. $122+-16 \mathrm{~ms} ; P<.01)$ and the QCI was close-to-significantly higher $(-31+-10 \%$ vs. $-21+-14 \% ; P=.09)$ in the LBBAP group.

\section{Discussion}

The main findings of this study were that narrower QRS complexes and better pacing outcomes (threshold and detection) were obtained at implantation and at three months using LBBAP in comparison to HBP.

The success rate was similar between LBBAP and HBP, which achieved comparable outcomes to those previously described in studies on each technique. ${ }^{8}$ No variable was related to a lower success rate with either technique. The presence of a previously implanted cardiac device negatively affected the success of the implant with both techniques and in the group as a whole, as previously reported. ${ }^{15}$ Problems related to the design and functioning were responsible for LBBAP failures, with four cases in which the interventricular septum could not be penetrated, and for HBP failures, with six cases in which the His electrogram was not recorded or the threshold was exceeded; in another three patients, HBP failure was due to the impossibility of narrowing the QRS duration. The percentage of lead-related complications was comparable to that observed by other authors ${ }^{16}$ and was higher with the HBP procedure.

To date, no study has directly compared the two techniques. Wang et al. ${ }^{17}$ published comparable results (for threshold and detection) to the present findings in 44 patients after HBP and in 8 after LBBAP, but they did not analyze differences between the techniques and did not compare data on QRS duration. In the present series, energy (threshold) requirements were decreased and the sensed R-wave amplitude was increased with LBBAP in comparison to HBP, both immediately post-implantation and at three months. The His bundle was separated from the membranous septum and insulated by thick fibers in a substantial proportion (32\%) of cases, ${ }^{18}$ signifying that high energy is needed to activate the conduction system at this site, a situation that does not arise in left branch pacing. The radiological exposure time was shorter with the LBBAP technique because it does not require a search for the His-bundle electrogram, which is essential in HBP.

Among the successful procedures, the paced QRS duration was shorter with LBBAP than with HBP, and the values for each technique were in line with previous reports. ${ }^{8}$ In cases with a narrow baseline QRS, 
the QRS interval lengthening after LBBAP was greater than after selective HBP and lesser than after nonselective HBP (Figure 4). Non-selective HBP gives rise to wider QRS intervals in comparison to selective HBP because it captures muscle cells that are closer to the His bundle stem, although this increased QRS duration has not proven to be clinically relevant. ${ }^{19}$ Any position in the RV endocardium (apex, outflow tract, septum) produces wider paced QRS complexes, explaining the not inconsiderable incidence of post-pacing dysfunction. For this reason, HBP or LBBAP is the procedure of choice in patients needing ABT, especially if a pacing percentage of $>20 \%$ is anticipated. According to our experience, LBBAP achieves the same success rate as HBP but with better pacing values and a lower radiological exposure time.

Outcomes were more favorable with LBBAP than with HBP in patients with intraventricular conduction disturbance. The anatomical localization of this disorder crucially influences the possibility of BBB correction. According to Upadhyay et al. ${ }^{20}$, the likelihood of a reversal using HBP is lower with a more distal localization of this anomaly. A more distal pacing was achieved with LBBAP (Figure 2) at the basal interventricular septum, where left branch fibers of the left branch are present in the subendocardium with a high degree of ramification, likely improving contact between the lead and the septal Purkinje system. ${ }^{6}$ In our patients with LBBB, a success rate of $100 \%$ was obtained with LBBAP and $66.7 \%$ with HBP was $(P<.1)$, although the techniques obtained a similar reduction in QRS width among successful cases. However, the percentage of cases with paced QRS of [?] 120 ms was close-to-significantly higher in the HBP group (75\% vs. $27.3 \%$; $P=.07)$.

Successful RBBB correction is also likely to depend on the anatomical site of the disorder, but scant data are available. Sharma et al. ${ }^{21}$ reported that HRB achieved a success rate of $95 \%$ (with no control group) in patients with RBBB and a baseline QRS of [?]120 ms and LVEF of [?] 50, reducing the mean QRS width from 158 to $127 \mathrm{~ms}$ and achieving correction in $78 \%$ of cases. In the present study, the success rate was 87.5\% with HBP and a similar reduction in QRS width was achieved (159 to $122 \mathrm{~ms}$ ), although it remained at [?]120 ms in $50 \%$ of the patients. In contrast, only one patient (9.1\%) in our LBBAP group had a paced QRS of $120 \mathrm{~ms}$ after the procedure. and they showed a greater reduction in the QRS complex $(P=.09)$ that reached statistical significance $(P<.05)$ in those with a baseline QRS of [?]150 ms.

The degree of narrowing of the QRS complex by conventional CRT is associated with a higher response rate and has been described as an independent predictor by some authors. ${ }^{14}$ In our series, LBBAP achieved a higher degree of narrowing in patients with a QRS duration of [?]130 ms than was obtained with HBP or with conventional CRT in patients with previous failure of HBP/ LBBAP pacing.

\section{Study limitations}

The main study limitations were the small sample size and the lack of randomization in the assignment to one or other technique, which were conducted during different time periods. Experience was first acquired with HBP, which may have influenced the reduced fluoroscopy time observed with LBBAP. A further limitation was the wide variety in the clinical situations of the patients, reducing the statistical power for comparisons between subgroups. Some differences that were close to significant may have reached statistical significance with a larger sample size; nevertheless, it proved adequate to detect statistically significant between-group differences in some outcomes. One study strength is that all procedures were conducted by one surgeon, avoiding inter-operator variability. In general, the results obtained for paced QRS width, threshold, and sensed R-wave amplitude were comparable to those published in studies with larger sample sizes. Randomized studies are warranted to compare these techniques in larger samples of patients in different clinical situations.

\section{Conclusions}

In comparison to HBP, LBBAP achieves a narrower paced QRS, lower threshold, improved R-wave detection, and shorter fluoroscopy time with a similar complication rate, and it offers a greater degree of narrowing in patients with wide baseline QRS.

Funding 
This research did not receive any specific grant from funding agencies in the public, commercial, or not-forprofit sectors.

\section{References}

Deshmukh P, Casavant DA, Romanyshyn M, Anderson K. Permanent, direct His-bundle pacing: A novel approach to cardiac pacing in patients with normal His-Purkinje activation. Circulation 2000;101:869-877.

Sharma PS, Dandamudi G, Naperkowski A, Oren JW, Storm RH, Ellenbogen KA, et al. Permanent Hisbundle pacing is feasible, safe, and superior to right ventricular pacing in routine clinical practice. Heart Rhythm 2015;12:305-312. https://doi.org/10.1016/j.hrthm.2014.10.021

Abdelrahman M, Subzposh FA, Beer D, Durr B, Naperkowski A, Sun H, et al. Clinical outcomes of His bundle pacing compared to right ventricular pacing. J Am Coll Cardiol 2018;71:2319-2330.

Barba-Pichardo R, Manovel Sanchez A, Fernandez-Gomez JM, Morina-Vazquez P, Venegas-Gamero J, Herrera-Carranza M. Ventricular resynchronization therapy by direct His-bundle pacing using an internal cardioverter defibrillator. Europace 2013;15:83-88.

Lustgarten DL, Crespo EM, Arkhipova-Jenkins I, Lobel R, Winget J, Koehler J et al. His-bundle pacing versus biventricular pacing in cardiac resynchronization therapy patients: A crossover design comparison. Heart Rhythm 2015;12:1548-1557.

Vijayaraman P, Naperkowski A, Subzposh FA, Abdelrahman M, Sharma PS, Oren JW, et al. Permanent His-bundle Pacing: Long-term Lead Performance and Clinical Outcomes. Heart Rhythm 2018;15:696-702.

Upadhyay GA, Cherian T, Shatz DY, Beaser AD, Aziz Z, Ozcan C, et al. Intracardiac delineation of septal conduction in left bundle-branch block patterns: Mechanistic evidence of left intrahisian block circumvented by His bundle pacing. Circulation 2019;139:1876-1888.

Li Y, Chen K, Dai Y, Li C, Sun Q, Chen R, et al. Left bundle branch pacing for symptomatic bradycardia: Implant success rate, safety, and pacing characteristics. Heart Rhythm 2019;16:1758-1765.

Hou X, Qian Z, Wang Y, Qiu Y, Chen X, Jiang H, et al. Feasibility and cardiac synchrony of permanent left bundle branch pacing through the interventricular septum. Europace 2019;21:1694-1702.

Vijayaraman P, Dandamudi G. How to perform permanent His bundle pacing: Tips and tricks. Pacing Clin Electrophysiol 2016;39:1298-1304.

Vijayaraman P, Dandamudi G, Zanon F, Sharma PS, Tung R, Huang W, et al. Permanent His bundle pacing: recommendations from a Multicenter His Bundle Pacing Collaborative His bundle Working Group for standardization of definitions, implant measurements, and follow-up. Heart Rhythm 2018;15:460-8.

Li X, Li H, Ma W, Ning X, Liang E, Pang K, et al. Permanent left bundle branch area pacing for atrioventricular block: Feasibility, safety, and acute effect. Heart Rhythm 2019;16:1766-1773

Upadhyay GA, Vijayaraman P, Nayak HM, Verma N, Dandamudi G, Sharma PS, et al. His Corrective Pacing or Biventricular Pacing for Cardiac Resynchronization in Heart Failure. J Am Coll Cardiol 2019;74:157-159.

Rickard J, Popovic Z, Verhaert D, Sraow D, Baranowski B, Martin DO, et al. The QRS narrowing index predicts reverse left ventricular remodeling following cardiac resynchronization therapy. Pacing Clin Electrophysiol 2011;34:604-611.

Dawson LP, Cadden J, Pol D, Wynn G, Gregg L, Kalman J, et al. Learning curve and initial experience with implementation of a His-bundle pacing program in an Australian setting. Heart Lung Circ 2020:in press.

Zanon F, Ellenbogen KA, Dandamudi G, Sharma PS, Huang W, Lustgarten DL, et al. Permanent His-bundle pacing: A systematic literature review and meta-analysis. Europace 2018;20:1819-1826. 
Wang S, Wu S, Xu L, Xiao F, Whinnett ZI, Vijayaraman P, et al. Feasibility and efficacy of His bundle pacing or left bundle pacing combined with atrioventricular node ablation in patients with persistent atrial fibrillation and implantable cardioverter-defibrillator therapy. J Am Heart Assoc 2019;8:e014253.

Kawashima, T., Sasaki, H. A macroscopic anatomical investigation of atrioventricular bundle locational variation relative to the membranous part of the ventricular septum in elderly human hearts. Surg Radiol Anat 2005;27:206-213.

Beer D, Sharma PS, Subzposh FA, Naperkowski A, Pietrasik GM, Durr B, et al. Clinical outcomes of selective versus nonselective His bundle pacing. JACC Clin Electrophysiol 2019;5:766-774.

Elizari MV. The normal variants in the left bundle branch system. J Electrocardiol 2017:50:389-399. https://doi.org/10.1016/j.jelectrocard.2017.03.004

Sharma PS, Naperkowski A, Bauch TD, Chan JYS, Arnold AD, Whinnett ZI, et al. Permanent His bundle pacing for cardiac resynchronization therapy in patients with heart failure and right bundle branch block. Circ Arrhythm Electrophysiol 2018;11:e06613.

Conflicts of interest. Manuel Molina-Lerma and Miguel Alvarez have received honoraria from Medtronic. All other authors list no disclosures.

Data availability: The data underlying this article will be shared on reasonable request to the corresponding author.

Tables

Table 1: Baseline characteristics of the patients

\begin{tabular}{lllll}
\hline Variable & Total group $(\mathrm{n}=87)$ & HBP group $(\mathrm{n}=45)$ & LBBAP group $(\mathrm{n}=42)$ & $P$ \\
Age, years & $76(64-81)$ & $75.5(62.5-82.5)$ & $76(64.2-81)$ & .7 \\
Sex male, n (\%) & $53(60.9)$ & $28(62.2)$ & $25(59.5)$ & .8 \\
HT, n (\%) & $67(77)$ & $40(89)$ & $27(64.3)$ & $<.01$ \\
DM, n (\%) & $37(42.5)$ & $21(46.7)$ & $16(38.1)$ & .42 \\
Heart disease, n (\%) & $46(53)$ & $18(40)$ & $28(66.7)$ & $<.05$ \\
LVEF, \% & $60(35-60)$ & $60(34.5-60)$ & $52.5(34.7-60)$ & .47 \\
Depressed LVEF, n (\%) & $38(43.7)$ & $17(37.8)$ & $21(50)$ & .25 \\
Dilated RA, n (\%) & $39(44.8)$ & $21(46.7)$ & $18(42.9)$ & .72 \\
Dilated LA, n (\%) & $67(77.3)$ & $34(75.6)$ & $33(78.6)$ & .74 \\
Previous device, n (\%) & $13(15)$ & $8(17.8)$ & $5(11.9)$ & .44 \\
Sinus atrial rhythm, n (\%) & $69(79.3)$ & $32(71.1)$ & $37(88.1)$ & .051 \\
PR interval, ms & $196(178-234)$ & $192(160-220)$ & $200(180-238)$ & .28 \\
BBB, n (\%) & $49(56)$ & $25(55.5)$ & $24(57.1)$ & .91 \\
QRS complex, ms & $145.5 \pm 44$ & $148.3 \pm 48$ & $142.5 \pm 38$ & .54 \\
Wide QRS complex, n (\%) & $55(63.2)$ & $28(62.2)$ & $27(64.3)$ & .84 \\
Therapy indication & & & & .25 \\
ABT, n (\%) & $51(58.6)$ & $29(64.4)$ & $22(52.4)$ & \\
CRT, n (\%) & $36(41.4)$ & $16(35.6)$ & $20(47.6)$ & \\
\hline
\end{tabular}

RA: right atrium; LA: left atrium; DM: diabetes mellitus; HT: hypertension (high blood pressure). Other abbreviations previously defined.

Table 2. Comparison of implantation variables and pacing parameters between groups

\begin{tabular}{llll}
\hline Variable & HBP $(\mathrm{n}=37)$ & LBBAP $(\mathrm{n}=40)$ & $P$ \\
Therapy indication & & & .48
\end{tabular}




\begin{tabular}{llll} 
ABT, n (\%) & $24(64.1)$ & $22(55)$ & \\
CRT, n (\%) & $13(35.9)$ & $18(45)$ & \\
Previous device, n (\%) & $6(15.4)$ & $4(10 \%)$ & .51 \\
Deflectable sheath, n (\%) & $7(18.9)$ & $3(7.5)$ & .18 \\
$>1$ de novo lead, n (\%) & $24(64.9)$ & $32(80)$ & .14 \\
FT, min & $17(11.5-29)$ & $10(7.25-13.95)$ & $<.001$ \\
Bundle branch block, n (\%) & $20(54.1)$ & $22(55)$ & .93 \\
Baseline QRS, ms & $143 \pm 45$ & $140 \pm 37$ & .64 \\
Paced QRS, ms & $117 \pm 19$ & $109 \pm 8.2$ & $<.05$ \\
QCI, \% & $-13.5 \pm 19.4$ & $-16.2 \pm 23.7$ & .6 \\
Wide baseline QRS, \% & $23(62.2)$ & $25(62.5)$ & .97 \\
Wide paced QRS, \% & $17(45.9)$ & $5(12.5)$ & .001 \\
Threshold at implantation, V & $1.5(0.75-2.1)$ & $0.5(0.5-0.74)$ & $<.001$ \\
Threshold at 3 months, V & $1.75(0.75-2.4)$ & $0.72(0.5-0.75)$ & $<.001$ \\
R-wave detection at implantation, mV & $4 \pm 3$ & $9.8 \pm 3.9$ & $<.001$ \\
R-wave detection at 3 months, mV & $4.9 \pm 2.9$ & $13.5 \pm 4.5$ & $<.001$ \\
Impedance at implantation, & $538 \pm 127$ & $670 \pm 94$ & $<.001$ \\
Impedance at 3 months, & $437 \pm 104$ & $560 \pm 135$ & $<.001$ \\
\hline
\end{tabular}

FT: Fluoroscopy Time; QCI: QRS Narrowing Index. Other abbreviations previously defined.

Table 3. Comparison between groups in patients with bundle branch block

\begin{tabular}{|c|c|c|c|}
\hline Overall group $(\mathrm{n}=50)$ & HBP & LBBAP & $\mathrm{P}$ \\
\hline Bundle branch block, $\mathrm{n}$ & 26 & 24 & .98 \\
\hline $\mathrm{RBBB}, \mathrm{n}(\%)$ & $14(53.8)$ & $13(54.2)$ & \\
\hline LBBB, n $(\%)$ & $12(46.2)$ & $11(45.8)$ & \\
\hline Overall success, n (\%) & $20(76.9 \%)$ & $22(91.7)$ & .25 \\
\hline RBBB success, n (\%) & $12(87.5)$ & $11(84.6)$ & 1 \\
\hline LBBB success, n (\%) & $8(66.7)$ & $11(100)$ & .09 \\
\hline Overall success $(\mathrm{n}=42)$ & HBP $(n=20)$ & LBBAP $(n=22)$ & $\mathrm{P}$ \\
\hline Baseline QRS, ms & $172 \pm 36$ & $164 \pm 19$ & .42 \\
\hline Paced QRS, ms & $124 \pm 20$ & $109 \pm 8$ & $<.01$ \\
\hline QCI, \% & $-26 \pm 14$ & $-33 \pm 8$ & .07 \\
\hline QRS [?] $120 \mathrm{~ms}$ after implantation, n (\%) & $12(60)$ & $4(18.2)$ & .01 \\
\hline LBBB success $(n=20)$ & HBP $(n=8)$ & $\operatorname{LBBAP}(n=11)$ & $\mathrm{P}$ \\
\hline Baseline QRS, ms & $190 \pm 39$ & $172 \pm 19$ & .27 \\
\hline Paced QRS, ms & $127 \pm 26$ & $112 \pm 9$ & .16 \\
\hline QCI, \% & $-32 \pm 13$ & $-34.8 \pm 4.8$ & .59 \\
\hline QRS [?] $120 \mathrm{~ms}$ after implantation, n (\%) & $6(75)$ & $3(27.3)$ & .07 \\
\hline RBBB success $(\mathrm{n}=32)$ & $\operatorname{HBP}(n=12)$ & $\operatorname{LBBAP}(n=11)$ & $\mathrm{P}$ \\
\hline Baseline QRS, ms & $159 \pm 30$ & $156 \pm 16$ & .73 \\
\hline Paced QRS, ms & $122 \pm 16$ & $107 \pm 7$ & $<.01$ \\
\hline QCI, \% & $-21 \pm 14$ & $-31 \pm 10$ & .09 \\
\hline QRS [?] $120 \mathrm{~ms}$ after implantation, n (\%) & $6(50)$ & $1(9.1)$ & .07 \\
\hline
\end{tabular}

LBBB: left bundle branch block; RBBB: right bundle branch block. Other abbreviations previously defined.

Figure legends

Figure 1. Precordial electrocardiogram leads. 
A: Sinus rhythm with atrioventricular block and narrow QRS. B: Same patient as A with non-selective HBP. C: Sinus rhythm with narrow QRS. D: Same patient as C with LBBAP. E: Sinus rhythm with LBBB. F: Same patient as E with resynchronization by HBP. G: Sinus rhythm with LBBB. H: Same patient as G with resynchronization by LBBAP.

Figure 2. Post-procedure imaging tests.

Chest X-ray. A: HBP; B: LBBAP. Transthoracic echocardiogram, long-axis 4-chamber view. C: lead placed in His bundle (white arrow); D: LBBAP lead penetrating interventricular septum (asterisk).

Figure 3: Progress of pacing parameters.

A: Pacing threshold in volts; B: Sensed R-wave amplitude in millivolts. At implantation (blue box) and at three months (red box).

Figure 4: QRS width analysis in patients with baseline QRS $<120 \mathrm{~ms}$.

Baseline QRS (blue box), paced QRS (green box) and QCI (orange box). NS-HBP: non-selective His bundle pacing; S-HBP: selective His bundle pacing.

Figure 5: QRS width analysis in patients with baseline QRS [?] $130 \mathrm{~ms}$.

Baseline QRS (blue box), paced QRS (green box) and QCI (orange box).

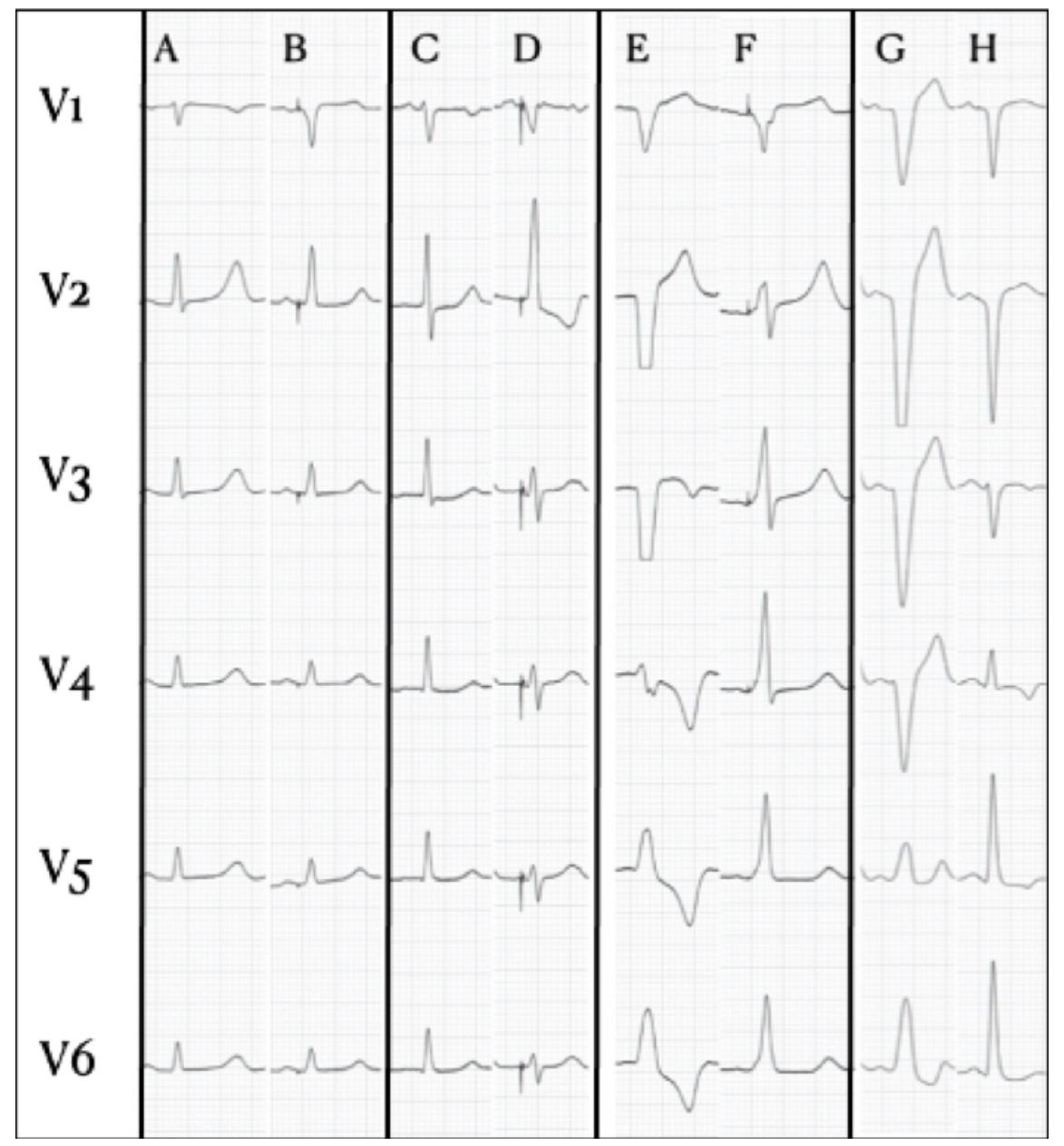



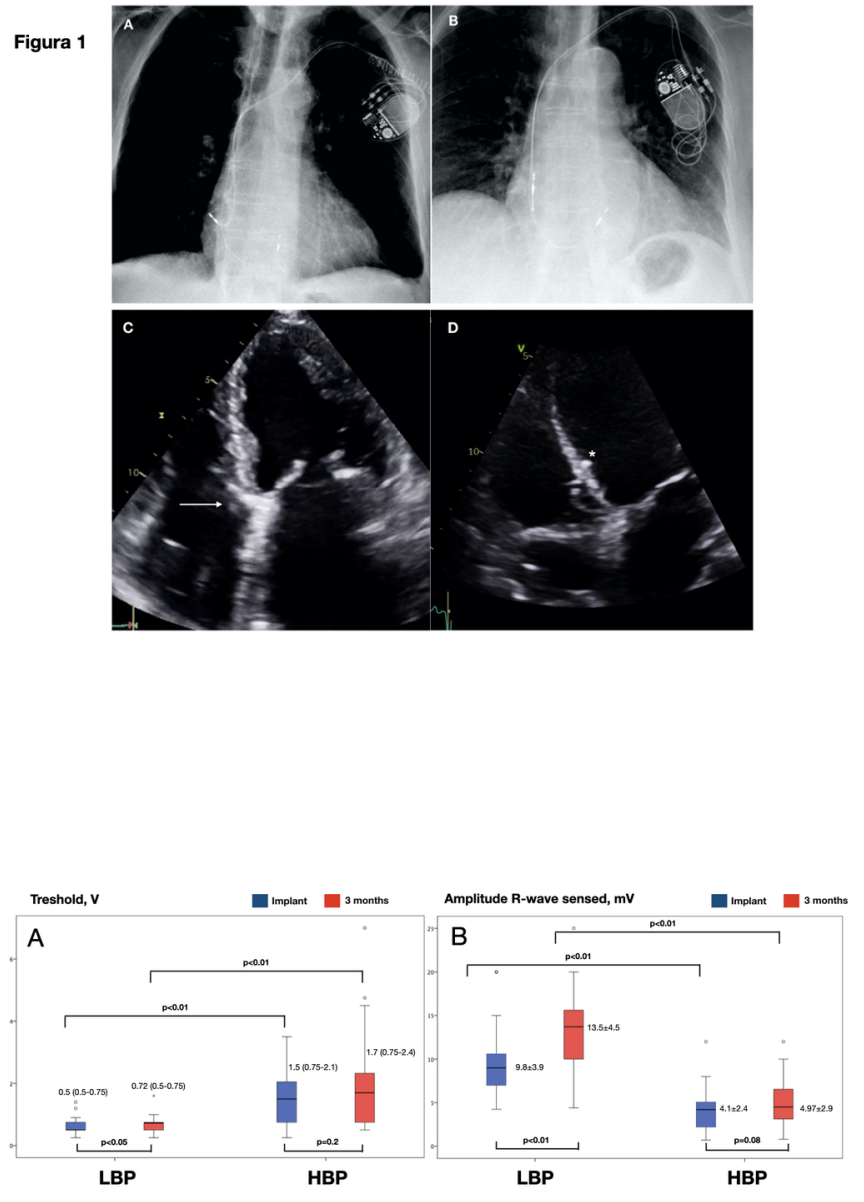

Baseline QRS (ms, mean) $\square$ Paced QRS (ms, mean) $\square$ QGI (\%, mean)

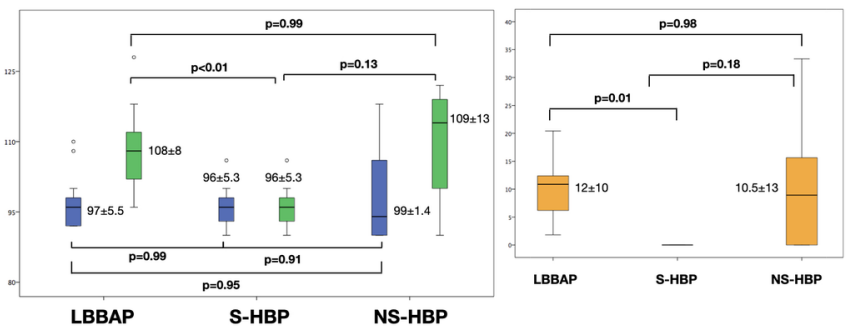




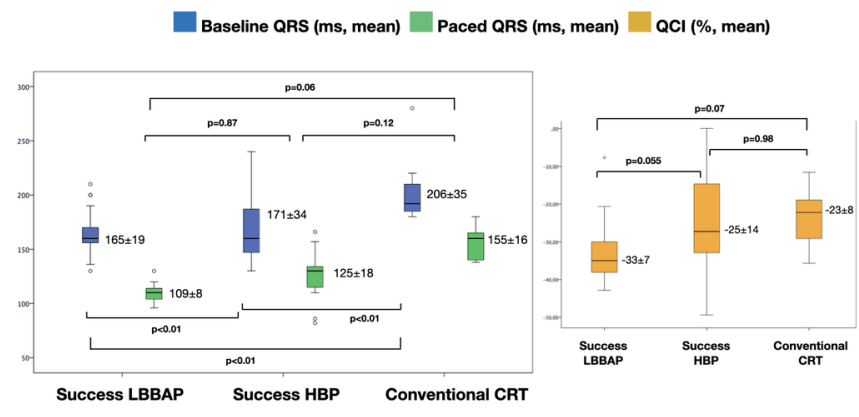

